\title{
ON THE ABSOLUTE CONVERGENCE OF LACUNARY FOURIER SERIES
}

\author{
J. R. PATADIA AND V. M. SHAH
}

\begin{abstract}
In 1958, P. B. Kennedy [2] proved a two-part theorem on the order of magnitude of the coefficients and the absolute convergence of lacunary Fourier series, and he conjectured that his conclusions remained valid under weaker conditions. The conjecture on the order of magnitude of the coefficients was established by $M$. and $\mathbf{S}$. I. Izumi [1]. In this note the second half of the conjecture, concerning absolute convergence, is deduced from a recent result of Patadia [3].
\end{abstract}

1. Introduction. Let

$$
\sum_{k=1}^{\infty}\left(a_{n_{k}} \cos n_{k} x+b_{n_{k}} \sin n_{k} x\right)
$$

be the Fourier series of a $2 \pi$-periodic function $f \in L[-\pi, \pi]$ with an infinity of gaps $\left(n_{k}, n_{k+1}\right)$, where $\left\{n_{k}\right\}(k \in \mathbf{N})$ is a strictly increasing sequence of natural numbers. Kennedy has proved the following theorem.

TheOREM [2, TheOREM II]. If $f \in \operatorname{Lip} \alpha(0<\alpha<1)$ in a subset $E$ of $[-\pi, \pi]$ of positive spread and if $\left\{n_{k}\right\}$ satisfies the gap condition

$$
\frac{n_{k+1}-n_{k}}{n_{k}^{\beta} \log n_{k}} \rightarrow \infty \quad \text { as } k \rightarrow \infty
$$

where $0<\beta<1$, then

(i) $a_{n}, b_{n}=O\left(n^{-\alpha \beta}\right)$, and

(ii) the Fourier series (1.1) of $f$ converges absolutely when $\alpha>\frac{1}{2}\left(\beta^{-1}-1\right)$.

Kennedy then conjectures that the conclusions of the theorem remain true if the factor $\log n_{k}$ in (1.2) is suppressed. The conjecture relating to part (i) of the theorem was proved in an even stronger form (involving the replacement of $E$ by a single point) by $\mathrm{M}$. and S. I. Izumi [1, Theorem 1]. The object of this note is to prove a similarly strengthened form of Kennedy's conjecture for part (ii) of his theorem.

We consider the gap condition

$$
\left(n_{k+1}-n_{k}\right)>C n_{k}^{\beta} k^{\theta} \quad(0<\beta<1, \theta \geqslant 0)
$$

where $C$ is a positive constant, and we prove the following theorem.

Received by the editors January 13, 1981.

AMS (MOS) subject classifications (1970). Primary 42A44.

Key words and phrases. Lacunary Fourier series, absolute convergence, modulus of continuity, Lipschitz condition. 
THEOREM. If $f \in \operatorname{Lip} \alpha(0<\alpha<1)$ at some point $x_{0} \in(-\pi, \pi)$ and if $\left\{n_{k}\right\}$ satisfies (1.3), then

$$
\sum_{k=1}^{\infty}\left(\left|a_{n_{k}}\right|^{r}+\left|b_{n_{k}}\right|^{r}\right)<\infty \quad(0<r \leqslant 1),
$$

when $\alpha \beta r+\alpha r \theta>(1-r / 2)(1-\beta)$.

REMARK. The particular case $r=1, \theta=0$ of the theorem provides verification of Kennedy's conjecture on the absolute convergence of the Fourier series (1.1), with the additional feature that the set of positive spread is replaced by a single point. The theorem also generalizes Theorem 2 in the paper [1] of M. and S. I. Izumi.

2. Proof of the theorem. We need the following lemma.

Lemma. Suppose that $\left\{n_{k}\right\}$ satisfies (1.3) and that $\delta$ is such that $0<\delta<$ $(1+\theta) /(1-\beta)$. Then there exists an integer $k_{0}$ and a real number $B$ such that $0<B<1$ and $n_{k}>B k^{\delta}$ for all $k \geqslant k_{0}$.

Proof. Since $\delta<(1+\theta) /(1-\beta)$ we get $\delta \beta+\theta>\delta-1$. Put $\delta \beta+\theta-\delta+1$ $=r$, so that $r>0$. Let $M$ be a positive integer such that $M>(1+\theta) /(1-\beta)$, and $k_{0}$ be a positive integer such that $C k_{0}^{r}>2^{M}$. We shall prove the lemma by induction.

Choose $B, 0<B<1$, such that $n_{k_{0}}>B k_{0}^{\delta}$.

Assume that $p$ is an integer such that $p \geqslant k_{0}$ and $n_{p}>B p^{\delta}$. Then, observing that $B<B^{\beta}$, we get by (1.3)

$$
\begin{aligned}
n_{p+1} & >n_{p}+C n_{p}^{\beta} p^{\theta}>B p^{\delta}+C B^{\beta} p^{\delta \beta+\theta} \\
& >B p^{\delta}+C B p^{\delta-1+r}=B p^{\delta}\left(1+C p^{r} / p\right)
\end{aligned}
$$

But

$$
\begin{aligned}
C p^{r} & \geqslant C k_{0}^{r}>2^{M}-1=\left(\begin{array}{c}
M \\
1
\end{array}\right)+\left(\begin{array}{c}
M \\
2
\end{array}\right)+\left(\begin{array}{c}
M \\
3
\end{array}\right)+\cdots+\left(\begin{array}{c}
M \\
M
\end{array}\right) \\
& >\left(\begin{array}{c}
M \\
1
\end{array}\right)+\left(\begin{array}{c}
M \\
2
\end{array}\right) \frac{1}{p}+\left(\begin{array}{c}
M \\
3
\end{array}\right) \frac{1}{p^{2}}+\cdots+\left(\begin{array}{c}
M \\
M
\end{array}\right) \frac{1}{p^{M-1}},
\end{aligned}
$$

and therefore

$$
\begin{aligned}
1+\frac{C p^{r}}{p} & >1+\frac{1}{p}\left[\left(\begin{array}{c}
M \\
1
\end{array}\right)+\left(\begin{array}{c}
M \\
2
\end{array}\right) \frac{1}{p}+\left(\begin{array}{c}
M \\
3
\end{array}\right) \frac{1}{p^{2}}+\cdots+\left(\begin{array}{c}
M \\
M
\end{array}\right) \frac{1}{p^{M-1}}\right] \\
& =(1+1 / p)^{M}>(1+1 / p)^{\delta} .
\end{aligned}
$$

Hence, by (2.1) and (2.2)

$$
n_{p+1}>B p^{\delta}(1+1 / p)^{\delta}=B(p+1)^{\delta}
$$

It then follows by induction that $n_{k}>B k^{\delta}$ for all $k \geqslant k_{0}$.

Proof OF THE THEOREM. We have $\alpha \beta r+\alpha r \theta>(1-r / 2)(1-\beta)$. Hence

$$
(1+\theta) /(1-\beta)>(2-r-2 \alpha r \theta) / 2 \alpha \beta r \text {. }
$$

Choose $\delta$ such that

$$
(1+\theta) /(1-\beta)>\delta>(2-r-2 \alpha r \theta) / 2 \alpha \beta r .
$$


Now,

(i) by the lemma $n_{k}>B k^{\delta}$ for all sufficiently large $k$;

(ii) since $f \in \operatorname{Lip} \alpha$ at $x_{0}, \omega\left(\delta, f, x_{0}\right)=O\left(\delta^{\alpha}\right)$, where

$$
\omega\left(\delta, f, x_{0}\right)=\sup _{0<h<\delta}\left\{\left|f\left(x_{0} \pm h\right)-f\left(x_{0}\right)\right|\right\} ;
$$

(iii) by (2.3), $\alpha \beta r \delta+\alpha r \theta+r / 2>1$.

Therefore we have

$$
\sum_{k=1}^{\infty} \frac{\left(\omega\left(A /\left(n_{k}^{\beta} k^{\theta}\right), f, x_{0}\right)\right)^{r}}{k^{r / 2}}=\sum_{k=1}^{\infty} \frac{O(1)}{\left(n_{k}^{\beta} k^{\theta}\right)^{\alpha r} k^{r / 2}}=\sum_{k=1}^{\infty} \frac{O(1)}{k^{\alpha \beta r \delta+\alpha r \theta+r / 2}}
$$

and the last series converges. The theorem now follows from [3, Theorem 1].

\section{REFERENCES}

1. M. Izumi and S. I. Izumi, On lacunary Fourier series, Proc. Japan Acad. Ser. A Math. Sci. 41 (1965), 648-651.

2. P. B. Kennedy, On the coefficients in certain Fourier series, J. London Math. Soc. (2) 33 (1958), 196-207.

3. J. R. Patadia, On the absolute convergence of lacunary Fourier series, Proc. Amer. Math. Soc. 71 (1978), 19-25.

Department of Mathematics, Faculty of Science, M.S. University Of Baroda, Baroda-390 002, INDIA 